\title{
Periodic patterns in a ring of delay-coupled oscillators
}

\author{
P. Perlikowski, ${ }^{1,2}$ S. Yanchuk, ${ }^{1}$ O. V. Popovych, ${ }^{3}$ and P. A. Tass ${ }^{3,4}$ \\ ${ }^{1}$ Institute of Mathematics, Humboldt University of Berlin, 10099 Berlin, Germany \\ ${ }^{2}$ Division of Dynamics, Technical University of Lodz, 90-924 Lodz, Poland \\ ${ }^{3}$ Institute of Neuroscience and Medicine-Neuromodulation (INM-7), Research Center Jülich, 52425 Jülich, Germany \\ ${ }^{4}$ Department of Stereotaxic and Functional Neurosurgery, University Hospital, 50924 Cologne, Germany
}

(Received 16 April 2010; revised manuscript received 24 August 2010; published 20 September 2010)

\begin{abstract}
We describe the appearance and stability of spatiotemporal periodic patterns (rotating waves) in unidirectional rings of coupled oscillators with delayed couplings. We show how delays in the coupling lead to the splitting of each rotating wave into several new ones. The appearance of rotating waves is mediated by the Hopf bifurcations of the symmetric equilibrium. We also conclude that the coupling delays can be effectively replaced by increasing the number of oscillators in the chain. The phenomena are shown for the Stuart-Landau oscillators as well as for the coupled FitzHugh-Nagumo systems modeling an ensemble of spiking neurons interacting via excitatory chemical synapses.
\end{abstract}

DOI: 10.1103/PhysRevE.82.036208

PACS number(s): 05.45.Xt, 02.30.Ks, 87.19.1j

\section{INTRODUCTION}

Networks of coupled dynamical systems [1] have become important models for studying the behavior of large complex systems. Such models allow us to investigate fundamental features of coupled systems of different kinds, ranging from neurons to lasers, from living organisms to climate systems [2-5]. Various types of collective behavior have been found and well understood including synchronization [6] and desynchronization [7], clustering [8-10], multistabilities of high degree [11-13], etc. Many different coupling schemes have been considered, including global all-to-all coupling $[8,9,14]$, local and nonlocal couplings $[11,15,16]$, as well as different kinds of random coupling supported by complex networks $[5,17,18]$ and network motifs [19]. Among them, networks with a ring topology, where locally coupled oscillators or oscillatory populations form a closed loop of signal transmission, appear to be relevant for many practical situations, e.g., in lasers and neuronal dynamics, and attracted a lot of attention in the past $[10,13,15,17,20-38]$. Ensembles with ring topology of unidirectionally coupled oscillators play an important role in the modeling of neuronal dynamics and information processing in neuroscience and medicine. Such networks are involved in the generation of stable periodic motor commands by central pattern generator of the nervous system controlling rhythmic locomotion in animals [39]. A ring-type topology is also present in the sequential feed-forward neuronal projections of a cortico-striatal-basal ganglia-thalamo-cortical motor loop relevant to the pathogenesis of Parkinson's disease [40].

Since in real systems the signal inevitably propagates from one oscillator to the next over a finite distance and with a finite speed, a non-negligible time delay in coupling naturally arises in such systems and plays an important role inducing some new phenomena and complex dynamics. This is the case, for example, in systems of coupled semiconductor lasers [19,41-47], neuronal networks [48-51], biological oscillators [20], etc. Already for a few coupled systems time delays impose challenging problems [16,19,41,45,52,53]. New phenomena emerge due to the inclusion of time delays in complex networks [54-57]. As related, for instance, to collective synchronized dynamics of delay-coupled oscillators, it has been found that delay may enhance or suppress the synchronization $[44,50,51,58,59]$. A number of methods based on delayed feedback have been designed to control synchronization utilizing either linear $[4,59]$ or nonlinear feedback [60-62]. A rather general delay-induced phenomenon seems to be the emergence of multistability of synchronized states as delay increases $[52,58,63]$.

This paper is devoted to the study of a unidirectionally coupled ring of oscillators interacting with time delays schematically illustrated in Fig. 1. We show that time delay in coupling may significantly influence the complexity of the collective dynamics by enhancing the multistability of synchronized spatiotemporal patterns. The latter, i.e., the spatiotemporal patterns or time-periodic solutions, including rotating waves, have been investigated by many authors. The existence and stability of phase-locked patterns and amplitude death states have been studied for a bidirectional ring of the Stuart-Landau oscillators [27]. Chaotic rotating waves have been reported for the Lorenz oscillators $[31,32]$ and nonlinear electronic Chua's circuits [28] unidirectionally coupled in rings and linear arrays. The case where the periodic oscillations in a ring are dumped and exist only as a transient has been addressed in Refs. [33,34]. On the other hand, persistent spatiotemporal patterns in rings of unidirectionally coupled oscillators have been analyzed in Refs. $[13,22,35-37]$. The transition from stationary to oscillatory behavior in the network is characterized by a complicated bifurcation scenario including the coexistence of multiple periodic orbits with different frequencies, spatial patterns, and modulation instabilities [13,38].

In this study we describe the appearance and stability of spatiotemporal periodic patterns (rotating waves), which emerge from a symmetric equilibrium as control parameters are varied. These patterns are similar to those appearing in systems without delay $[13,22,23]$. The main result of the paper is to show how the inclusion of additional delays in the coupling leads to the splitting of each rotating wave into several new ones. The appearance of additional rotating waves is mediated by the Hopf bifurcations of the origin, 


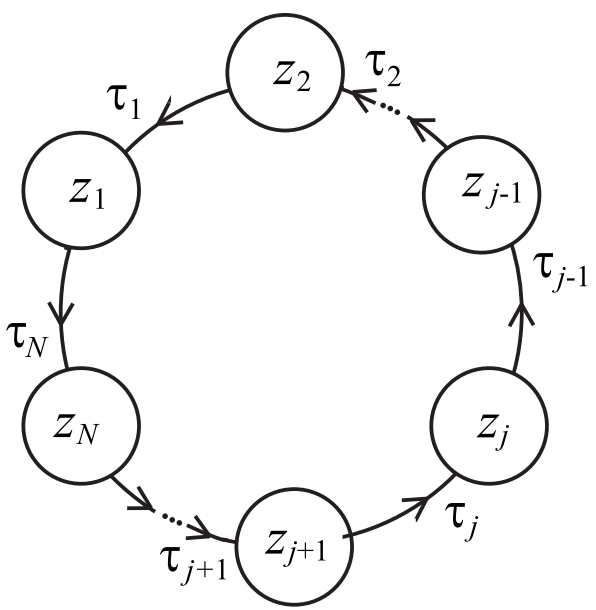

FIG. 1. Ring of unidirectionally coupled oscillators. $z_{j}$ are phase variables of individual oscillators and $\tau_{j}$ are coupling delays. Arrows denote coupling direction.

whose frequency is independent of the round trip delay time $T$ and the number of oscillators $N$ in the ring. Moreover, we show that the whole family of rotating waves has a very similar description in any of the following asymptotic cases: large number of oscillators $N$, large round trip time $T$, or both. We conclude that the coupling delays can effectively be replaced by increasing the number of oscillators in the chain. In particular, the rotating waves as well as their stability in a ring of few oscillators (or even one oscillator with a delayed feedback) have similar properties as the rotating waves in a ring of $N$ oscillators without delay provided $N$ is sufficiently large.

We consider two models: a paradigmatic model of the coupled Stuart-Landau oscillators in the first part of the paper (Secs. II and III) and a more realistic FitzHugh-Nagumo (FHN) neuronal systems $[64,65]$ interacting via excitatory chemical synapses in the second part (Sec. IV). The StuartLandau oscillator describes a normal form of a supercritical Hopf bifurcation, whereas the FitzHugh-Nagumo system belongs to a class of most useful and widely used models of spiking neurons [66]. The paper is organized as follows. In Sec. II we study the stability and spectrum of the symmetric equilibrium with respect to the control parameters $\alpha$ (natural amplitudes of the oscillators), $T$, and $N$. In Sec. III, we study the appearance of multiple periodic patterns as the equilibrium is destabilized through the Hopf bifurcations. In Sec. III B, we study the stability of these patterns and analyze the cases with large round trip time $T$ or large number of oscillators $N$. In Sec. IV we consider a system of unidirectionally coupled FHN oscillators and demonstrate that the phenomena observed in the previous sections for the coupled StuartLandau limit-cycle oscillators can be found for a ring of delay-coupled spiking neurons. Final conclusions are presented in Sec. V.

\section{STABILITY AND SPECTRUM OF THE SYMMETRIC EQUILIBRIUM}

The ring of the delayed unidirectionally coupled dissipative Stuart-Landau oscillators [14] has the following form:

$$
\dot{z}_{j}(t)=(\alpha+i \beta) z_{j}(t)-z_{j}(t)\left|z_{j}(t)\right|^{2}+z_{j+1}\left(t-\tau_{j}\right),
$$

where $1 \leq j \leq N, z_{j} \in \mathrm{C}$ are complex variables for individual oscillators, $\alpha$ and $\beta$ are real parameters, and $\tau_{j}>0$ are time delays of the coupling (see Fig. 1). The ring structure implies $z_{1} \equiv z_{N+1}$. The purpose of this paper is to investigate the effect of the coupling delays on the dynamics. Apart from the natural control parameter of the individual dynamics $\alpha$, the following important control parameters appear in this setup: the round trip time delay $T=\tau_{1}+\tau_{2}+\cdots+\tau_{N}$ and the number of oscillators $N$ in the ring. In particular, the cases with large number of oscillators $N$ or/and large round trip delay $T$ are investigated in detail.

Before proceeding to the analysis of the model, let us note that the delays in the coupling can be assumed to be identical without loss of generality. Indeed, given any distribution of delays $\tau_{1}, \tau_{2}, \ldots, \tau_{N}$, the following change of variables [38]

$$
\begin{gathered}
v_{N}(t)=z_{N}(t), \\
v_{N-1}(t)=z_{N-1}\left[t-\left(\tau-\tau_{N-1}\right)\right], \\
v_{N-2}(t)=z_{N-2}\left[t-\left(2 \tau-\tau_{N-1}-\tau_{N-2}\right)\right], \\
\cdots \\
v_{1}(t)=z_{1}\left[t-\left(\tau_{N}-\tau\right)\right],
\end{gathered}
$$

with $\tau=T / N$ leads to the same system as in Eq. (1) but with identical time delays $\tau$ with respect to $v_{j}(t)$. Therefore, we assume in the following that $\tau_{j}=\tau=T / N$. The results, which are obtained for identical delays, can be used for systems with dispersed delay times in an obvious way using transformation (2). Additional comments on this are given in Sec. V.

\section{A. Properties of the spectrum}

The symmetric equilibrium at the origin $z_{1}=\cdots=z_{N}=0$ plays an important role in the development of the nonstationary dynamics in system (1). In particular, as we show in Sec. III, periodic patterns emerge from this equilibrium as some bifurcation parameter is varied, similar to [13]. In order to study the corresponding bifurcations, in this section we investigate the eigenvalues of this equilibrium.

The stability of the origin can be studied by linearizing Eq. (1),

$$
\dot{z}_{j}(t)=(\alpha+i \beta) z_{j}(t)+z_{j+1}(t-\tau) .
$$

Linearized system (3) can be diagonalized using the methods described in $[13,67]$. As a result of such a diagonalization, instead of one high-dimensional coupled system (3) we obtain a set of $N$ uncoupled systems of lower dimension. Formally, it can be achieved by the following discrete Fourier transformation $z_{j}(t)=\sum_{l=1}^{N} e^{i \varphi_{l} j} w_{l}(t)$, where $\varphi_{l}=2 \pi l / N$. Applying this transformation to system (3), we obtain the following set of independent systems:

$$
w_{l}^{\prime}(t)=(\alpha+i \beta) w_{l}(t)+e^{i \varphi_{l}} w_{l}(t-\tau), \quad 1 \leq l \leq N .
$$

Obviously, Eq. (4) determines the stability of the coupling mode $\varphi_{l}, 1 \leq l \leq N$. The stability of each of these modes can 

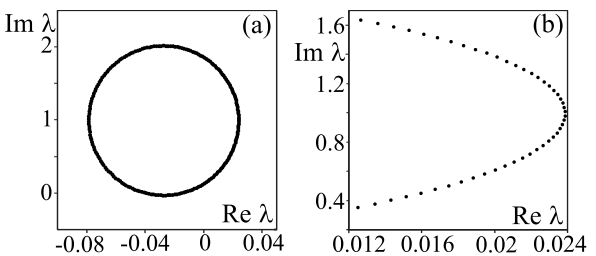

FIG. 2. Spectrum of the origin in the array of delay-coupled $N$ $=200$ oscillators. The round trip time is $T=10$, which corresponds to a small delay $\tau=0.05$ between the neighboring oscillators. The spectrum is approximated by expression (8). (a) Whole spectrum; (b) zoom of the eigenvalues with the largest real parts. $\alpha=-0.99$ and $\beta=1$.

be studied by the following characteristic equation:

$$
\lambda=(\alpha+i \beta)+e^{i \varphi_{l}-\lambda \tau},
$$

which is obtained by the substitution $w_{l}(t)=e^{\lambda t} w_{0 l}$. Note that the characteristic equations for different coupling modes $\varphi_{l}$ differ only by the phase factor $e^{i \varphi_{l}}$. If the number of oscillators is large, the values of $\varphi_{l}$ densely fill the interval from 0 to $2 \pi$ and one can approximate the phase $\varphi_{l}$ by some continuous parameter $0 \leq \varphi \leq 2 \pi$. Due to this fact we omit the index $l$ below,

$$
\lambda=(\alpha+i \beta)+e^{i \varphi-\lambda \tau}
$$

Equation (6) can formally be solved using the Lambert function $[68,69]$,

$$
\lambda=\Lambda_{\tau}(\varphi)=(\alpha+i \beta)+\frac{1}{\tau} W\left(\tau e^{i \varphi-(\alpha+i \beta) \tau}\right) .
$$

The following asymptotic cases can be studied analytically:

Case 1. The interaction delay between oscillators is small $\tau \ll 1$.

Case 2. The interaction delay between oscillators is larger than the other characteristic time scales.

In the first case, one can neglect the delay and obtain the following set of eigenvalues:

$$
\Lambda_{0}(\varphi)=(\alpha+i \beta)+e^{i \varphi}, \quad \varphi=\varphi_{l}, \quad 1 \leq l \leq N,
$$

which coincides with the spectrum for the ring of coupled oscillators without delay [13] (Fig. 2).

The situation becomes more complicated as the delay $\tau$ increases. In particular, for each fixed coupling mode $\varphi_{l}$, an infinite number of additional eigenvalues becomes relevant, which can be associated with the "delay modes." For large delay, the pseudocontinuous spectrum approach $[56,69,70]$ can be used. In order to obtain an approximation for the spectrum in this case, one should formally substitute $\lambda=i \omega$ $+\gamma / \tau$ in the characteristic Eq. (6). As a first-order approximation, we obtain the equation

$$
i \omega=(\alpha+i \beta)+e^{-\gamma+i(\varphi-\omega \tau)},
$$

which can be solved with respect to $\gamma(\omega)$,
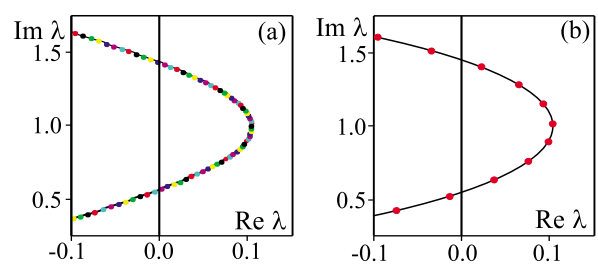

FIG. 3. (Color online) Spectrum of eigenvalues for $N=7$ delaycoupled Stuart-Landau oscillators with the round trip delay time $T$ $=350$. (a) Eigenvalues, associated with different coupling modes $\varphi_{l}=\frac{2 \pi}{N} l$ [see Eq. (5)], are shown in different colors; (b) only the spectrum for the mode $\varphi_{1}$ is shown. Other parameters: $\alpha=-0.99$ and $\beta=1$.

$$
\gamma(\omega)=-\frac{1}{2} \ln \left[\alpha^{2}+(\beta-\omega)^{2}\right] .
$$

The set of eigenvalues for large delay can be approximated by the following continuous curve $[56,69,70]$ :

$$
\lambda=\Lambda_{\tau \gg 1}(\omega)=\frac{\gamma(\omega)}{\tau}+i \omega,
$$

which is parametrized by $\omega$. It is important to observe that the curve $\Lambda_{\tau \gg 1}(\omega)$ does not depend on the coupling mode $\varphi$. This implies that the spectrum has the same asymptotic representation (9) for all coupling modes $\varphi_{l}$. As a result, the stability of each coupling mode is the same for sufficiently large delay. Figure 3(a) illustrates the whole spectrum of the eigenvalues and Fig. 3(b) shows only the eigenvalues, which are associated with one coupling mode. Even though the actual spectrum is discrete, curve (9) gives a reasonable approximation since the distances between the neighboring points of the spectrum are known $[56,70]$ to be small and proportional to $1 /(\tau N)$.

Finally note that in the case $\alpha>0$ and large $\tau$, a strongly unstable eigenvalue exists [56,69], which is close to $\alpha+i \beta$. This makes the system already unstable for all $\alpha>0$, and no interesting bifurcation scenarios occur.

\section{B. Conditions for the Hopf bifurcations and the Hopf frequencies}

The destabilization of the symmetric equilibrium occurs through the Hopf bifurcations. The condition for the Hopf bifurcations can be obtained by substituting $\Lambda=i \Omega_{H}$ into the characteristic equation

$$
i \Omega_{H}=(\alpha+i \beta)+e^{i \varphi-i \Omega_{H} \tau},
$$

where $\Omega_{H}$ is the Hopf frequency. Equation. (10) can be solved with respect to the Hopf frequency

$$
\left(\Omega_{H}-\beta\right)^{2}+\alpha^{2}=1
$$

or

$$
\Omega_{H}=\beta \pm \sqrt{1-\alpha^{2}}
$$

after excluding the unknown phase argument $\varphi-\Omega_{H} \tau$. Equation (12) shows that the Hopf frequencies are independent of the number $N$ of oscillators and the delay $\tau$ for given parameters of individual oscillators $\alpha$ and $\beta$. 


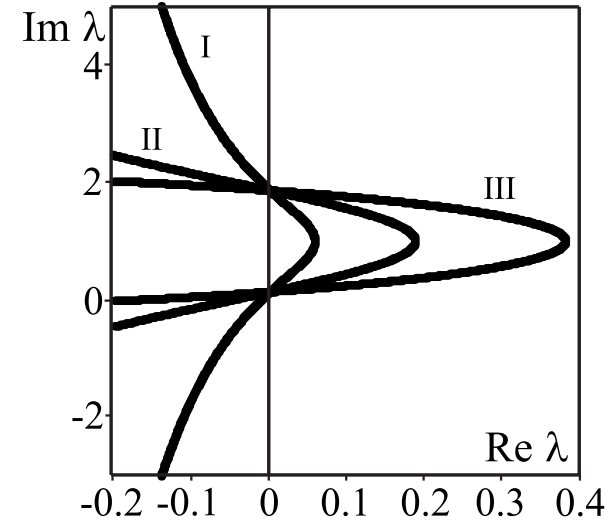

FIG. 4. A part of the spectrum, corresponding to the eigenvalues with the largest real parts (critical eigenvalues). The spectrum corresponds (i) to $N=100$, (ii) to $N=500$, and (iii) to $N=3000$. Although the eigenvalues come closer to the imaginary axis and scale as $1 / \tau$ with an increase of $\tau=T / N$, the spectrum crosses the imaginary axis at the same Hopf points $i \Omega_{H}$. The other parameters read $\alpha=-0.99, \beta=1$, and $T=1000$ and are fixed.

Note that the asymptotic approximations (8) and (9) of the spectrum preserve this property. More precisely, the Hopf frequencies, which can be obtained from the approximated spectra (8) and (9), coincide with the exact frequencies given in Eq. (11).

The illustration in Fig. 4 shows the spectra for different values of $N$ with some fixed $\alpha, \beta$, and $T$. One can observe that all spectra cross the imaginary axis at the same Hopf points $i \Omega_{H}$ as it is predicted by the theory. The additional effect is that the eigenvalues come closer to the imaginary axis as the delay $\tau$ increases and their real part scales as $1 / \tau$ [see Eq. (9)]. Yet this effect influences neither the Hopf frequency nor the destabilization threshold.

Figure 5 illustrates how the whole spectrum changes as the number of oscillators is changed. More precisely, the parameters $\alpha=-0.5, \beta=1$, and $T=100$ are fixed, and $N$ is decreased from 1000 to 10 (this corresponds to an increase of $\tau$ from 0.1 to 10 ). As the number of oscillators decreases, the spectrum transforms from that shown in Fig. 5(a), which is typical for lattices of coupled oscillators without delay, to the spectrum shown in Fig. 5(f), which is typical for systems with large delay.

\section{Hopf curves and stability domains with respect to parameters}

In the case, when the number of oscillators or delay time is large, the conditions for stability of the origin can be written using the asymptotic spectra. More exactly, in the case of large $N$ and small delay, the stability condition is $\operatorname{Re}\left[\Lambda_{0}(\varphi)\right]<0$, i.e.,

$$
\operatorname{Re}\left[(\alpha+i \beta)+e^{i \varphi}\right]<0, \quad 0 \leq \varphi \leq 2 \pi
$$

while in the case of a large delay $\tau$ ( $N$ can attain any positive integer value), the stability condition is $\operatorname{Re}\left[\Lambda_{\tau \gg 1}(\omega)\right]<0$, i.e.,
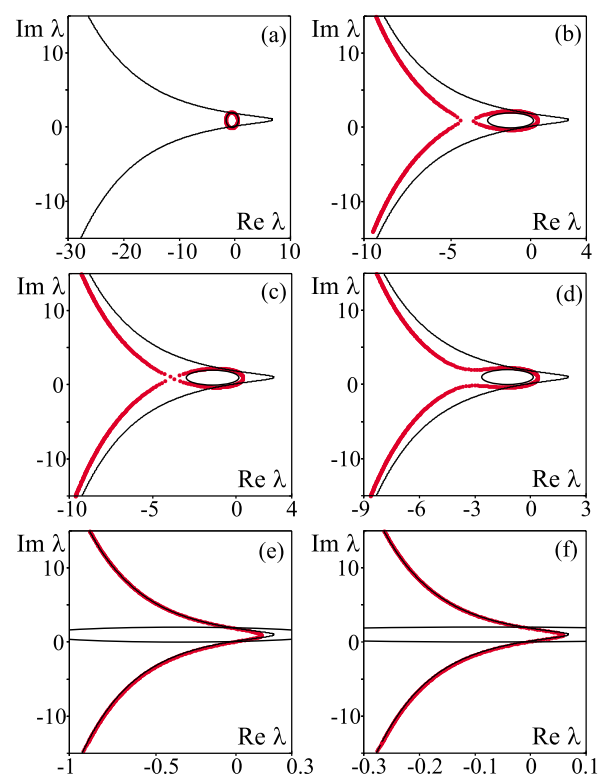

FIG. 5. (Color online) Change of the spectrum as $N$ is changed with fixed round trip $T$. The spectrum (bold red points) continuously transforms from the typical lattice spectrum in (a) to the typical large delay spectrum in (f). Parameter values: $\alpha=-0.5, \beta=1$, $T=100$, (a) $N=1000$, (b) $N=320$, (c) $N=318$, (d) $N=300$, (e) $N$ $=30$, and (f) $N=10$. Thin black lines show the positions of asymptotic spectra (8) and (9) for large $N$ and large $T$ limits.

$$
\operatorname{Re}\left[\frac{\gamma(\omega)}{\tau}+i \omega\right]<0, \quad \omega \in \mathbb{R}
$$

It is remarkable that both cases yield the same stability condition

$$
\alpha<-1
$$

This simple condition holds as soon as $N$ is large or $\tau$ is large. In the case, when $N$ or $\tau$ admits intermediate values, the stability of the origin can still be achieved for $\alpha>-1$. The explanation for this effect is the following: although the continuous asymptotic spectra (9) and (8) are unstable for $\alpha>-1$, the positions of the eigenvalues on the spectral lines may "miss" the unstable parts of the curves (similar to [69]).

Let us consider the exact characteristic Eq. (6) and obtain the curves of the Hopf bifurcations in the parameter space. From Hopf condition (10) we obtain

$$
\arg \left(-\alpha-i \beta+i \Omega_{H}\right)=\varphi-\Omega_{H} \tau_{H}
$$

Taking into account that $\varphi=\varphi_{l}=2 \pi l / N$ and $\tau=T / N$ we obtain

$$
\arg \left(-\alpha-i \beta+i \Omega_{H}\right)=\frac{2 \pi l}{N}-\Omega_{H} \frac{T}{N} .
$$

Let us choose $N$ and $T$ to be our control parameters. Then, the representation for the lines of the Hopf bifurcations in the parametric plane $(N, T)$ reads 


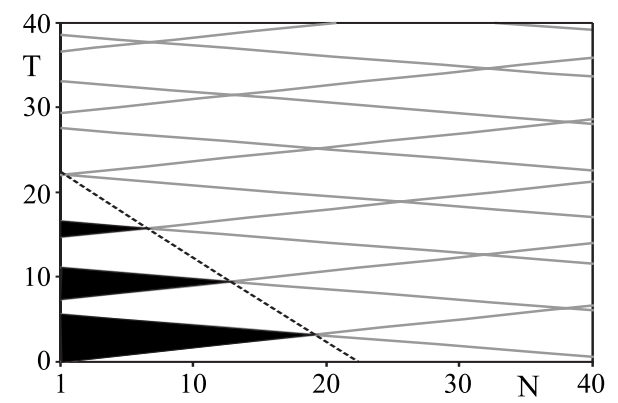

FIG. 6. Hopf bifurcation curves are shown in gray. The origin is stable for the parameter values from the black areas. The dashed black line is the stability boundary given by Eq. (16). $\alpha=-0.99$ and $\beta=1$.

$$
N=\frac{2 \pi l-\Omega_{H} T}{\arg \left(-\alpha-i \beta+i \Omega_{H}\right)}, \quad l=0, \pm 1, \pm 2, \ldots .
$$

Here, $\Omega_{H}$ is given by Eq. (12). Figure 6 shows a twoparameter diagram for $\alpha=-0.99$ and $\beta=1$, where the Hopf bifurcation lines are shown. The shaded areas in the bifurcation diagram denote the domains where the origin is stable. The boundary of this domain denotes the first Hopf bifurcation, which leads to the initial destabilization of the origin.

As predicted by the theory, the domains of stability disappear as $T$ or $N$ increases. Such a size limitation can be estimated quantitatively by finding the line in the parameter space, which connects the peaks of the stability domains (see Fig. 6). Each peak of the stability domain corresponds to the parameter value, for which a double Hopf bifurcation occurs. The condition for the double Hopf bifurcation is given by

$$
\begin{aligned}
& i \Omega_{H 1}=(\alpha+i \beta)+e^{i \varphi_{1}-i \Omega_{H 1} \tau}, \\
& i \Omega_{H 2}=(\alpha+i \beta)+e^{i \varphi_{2}-i \Omega_{H 2} \tau},
\end{aligned}
$$

where $\Omega_{H 1,2}=-\beta \pm \sqrt{1-\alpha^{2}}$ and $\varphi_{1}-\varphi_{2}=\frac{2 \pi}{N}$. If we denote $\chi(\alpha)=\arg \left(-\alpha+i \sqrt{1-\alpha^{2}}\right)$, we obtain from Eqs. (14) and (15)

$$
\begin{gathered}
\varphi_{2}+\frac{2 \pi}{N}-\beta \tau-\sqrt{1-\alpha^{2}} \tau=\chi(\alpha), \\
\varphi_{2}-\beta \tau+\sqrt{1-\alpha^{2}} \tau=-\chi(\alpha) .
\end{gathered}
$$

Excluding $\varphi_{2}$ and substituting $\tau=\frac{T}{N}$, we obtain

$$
\frac{2 \pi}{N}-2 \sqrt{1-\alpha^{2}} \frac{T}{N}=2 \chi(\alpha)
$$

from which we finally get

$$
T \sqrt{1-\alpha^{2}}+\chi(\alpha) N=\pi .
$$

The obtained Eq. (16) shows the boundary of the possible stability domain of the origin. As soon as $T \sqrt{1-\alpha^{2}}+\chi(\alpha) N$ $>\pi$, a stabilization is not possible. Although condition (16) is independent of $\beta$, exact positions of the stability domains (black regions in Fig. 6) do depend on the value of $\beta$.

\section{EMERGING PERIODIC PATTERNS}

In this section we describe emerging periodic solutions of system (1). We derive a relation between the number of the periodic solution branches and the coupling time delay $\tau$. We show that these periodic solutions correspond to periodic patterns emerging with increasing $N$ or/and $\tau$. In the second part of this section we analyze the stability of the periodic solutions (respectively, patterns) and compare different cases: coupled oscillators without delay, single oscillator with delay, and delayed coupled oscillators. The words "pattern" and "solution" are used interchangeably below, though the former one underlines the spatial structure and the latter one the fact that they are resulting from the dynamics of system (1).

\section{A. Shape of periodic patterns}

Due to the spatiotemporal symmetries, system (1) has periodic solutions in the form of rotating waves (similar to those seen in [13]),

$$
z_{j}(t)=a e^{i \varphi_{l} j+i \omega t},
$$

where $\varphi_{l}=\frac{2 \pi}{N} l$ and $a$ and $\omega$ are the unknown amplitude and frequency of the periodic solutions, respectively. After substituting Eq. (17) to Eq. (1) we obtain the following equation:

$$
i \omega=\alpha+i \beta-a^{2}+e^{i\left(\varphi_{l}-\omega \tau\right)},
$$

which leads to the following system:

$$
\begin{gathered}
a=\sqrt{\alpha+\cos \left(\varphi_{l}-\omega \tau\right),} \\
\omega=\beta+\sin \left(\varphi_{l}-\omega \tau\right)
\end{gathered}
$$

for the amplitude and frequency of a periodic solution. Let us denote the solutions of Eqs. (19) and (20) as $a_{l k}$ and $\omega_{l k}$. We number now the solutions with two indices $l$ and $k$ since for each fixed $l$, Eq. (20) has multiple solutions, the number of which increases with the delay. From Eq. (20) we see that $\beta-1 \leq \omega \leq \beta+1$.

Each periodic solution originates in a Hopf bifurcation [see Eq. (12)] of the symmetric equilibrium. The number of bifurcations grows with an increase of the number of oscillators as well as with increase of the time delay in the coupling. In Figs. 7(a) and 7(b) we show branches of periodic solutions for $N=11, \tau=0$ and $N=1, \tau=15$ versus control parameter $\alpha$. In these two cases the number of periodic solutions is the same. In Fig. 7(c) we illustrate how the number of solutions of Eq. (20) grows due to the increase of the time delay $\tau$ in the given range of $\beta$ [see Eq. (17)].

Figure 8 illustrates the spatiotemporal structure of different patterns. The upper part of the figure shows the patterns, which correspond to the coupling modes with $l=0, \pm 1$ and small delay. With an increase of the delay, each pattern gives rise to multiple additional delay-induced patterns (numbered by $k$ ) as illustrated in the lower figure for $l=0$.

\section{B. Stability of patterns}

The stability of the obtained periodic patterns can be described by the spectrum of the Floquet multipliers. Even 

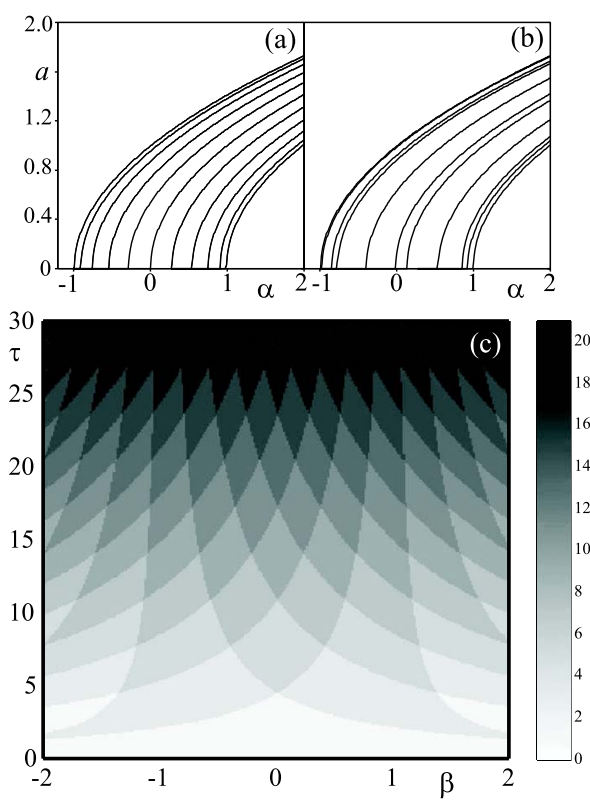

FIG. 7. (Color online) Families of periodic solutions for (a) ring of $N=11$ coupled oscillators without delay [see Eq. (17)] and (b) a single delayed oscillator with $\tau=15$ [see Eq. (24)]. The same number of branches is present in both cases. In (c) the number of periodic solutions is shown in gray as a function of two parameters: $\beta$ and the time delay $\tau$ for a single oscillator with the delayed feedback.

though the main purpose of this paper is to study the ring of delay-coupled oscillators, in what follows we compare three cases: a single oscillator with delayed feedback, a ring of unidirectionally coupled oscillators without time delay, and a ring of unidirectionally delay-coupled systems. We will show not only some remarkable similarities of the families of periodic solutions in all three cases but also important differences.

In order to determine the stability of the periodic patterns [Eq. (17)], one has to linearize the equations of motion [Eq. (1)] around the corresponding solutions. Before doing such a linearization, let us transform system (17) with the following change of coordinates (i.e., a corotating frame):

$$
z_{j}(t)=a_{l k} e^{i \varphi_{l} j+i \omega_{l k} t}\left[x_{j}(t)+i y_{j}(t)\right],
$$

where $x_{j}$ and $y_{j}$ are new real coordinates. With respect to the new coordinates, the system has the following form:

$$
\begin{aligned}
x_{j}^{\prime}= & \left(a_{l k}^{2}-\cos \chi_{l k}\right) x_{j}-y_{j} \sin \chi_{l k}-a_{l k}^{2} x_{j}\left(x_{j}^{2}+y_{j}^{2}\right) \\
& +x_{j+1}(t-\tau) \cos \chi-y_{j+1}(t-\tau) \sin \chi_{l k}, \\
y_{j}^{\prime}= & -x_{j} \sin \chi_{l k}+\left(a_{k}^{2}-\cos \chi_{l k}\right) y_{j}-a_{l k}^{2} y_{j}\left(x_{j}^{2}+y_{j}^{2}\right) \\
& +x_{j+1}(t-\tau) \sin \chi_{l k}+y_{j+1}(t-\tau) \cos \chi_{l k},
\end{aligned}
$$

and periodic solution (17) is transformed into the family of equilibria $x_{j}+i y_{j}=e^{i \kappa}, \kappa \in \mathbb{R}$. Here, we denote $\chi_{l k}=\varphi_{l}-\tau \omega_{l k}$. In order to find its stability, it is necessary to linearize around any of this equilibria since all of them have the same stability properties. We choose $x_{j}=y_{j}=1 / \sqrt{2}$. The corresponding linearized system reads in vector form (a)
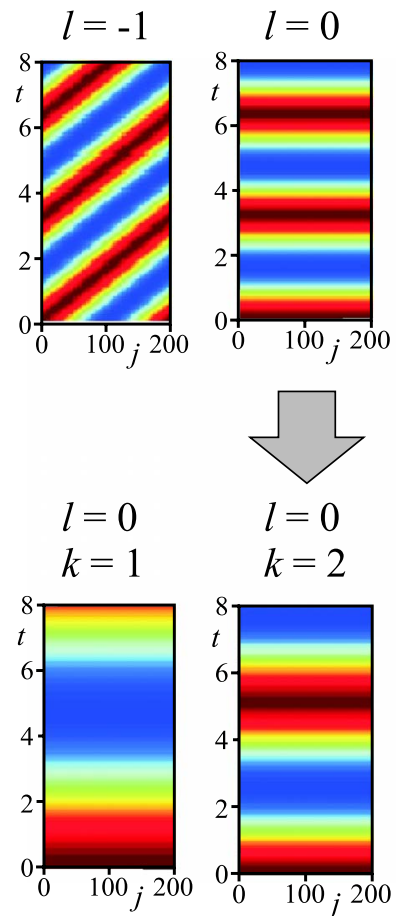

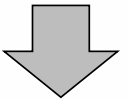

(b)

(c)

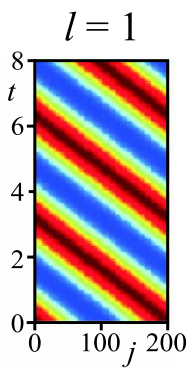

$l=0$

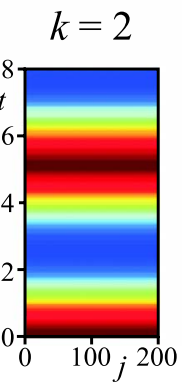

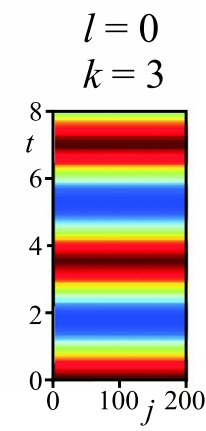

FIG. 8. (Color online) Spatiotemporal patterns for some of bifurcating time-periodic solution (17) corresponding to different values of $l$ (coupling modes). In (a)-(c) we show patterns in a ring of coupled oscillators where a particular value of $l$ corresponds to a single frequency $\omega_{l 1}$ and an amplitude $a_{l 1}$. As the delay increases, new frequencies $\omega_{01}, \omega_{02}$, and $\omega_{03}$ appear (as well as $\omega_{l 1}, \omega_{l 2}, \ldots$ ), which correspond to additional delay modes. The lower panel illustrates new delay-induced patterns for $l=0$ and $\tau=10$ [see also Fig. 7(c)]. $\alpha=-0.5, N=200$, and $\beta=1$.

$$
\left[\begin{array}{c}
\delta x_{j}^{\prime} \\
\delta y_{j}^{\prime}
\end{array}\right]=-\left(C_{l k}+a_{l k}^{2}\left[\begin{array}{cc}
1 & 1 \\
1 & 1
\end{array}\right]\right)\left[\begin{array}{l}
\delta x_{j} \\
\delta y_{j}
\end{array}\right]+C_{l k}\left[\begin{array}{l}
\delta x_{j+1}(t-\tau) \\
\delta y_{j+1}(t-\tau)
\end{array}\right],
$$

where

$$
C_{l k}=\left[\begin{array}{cc}
\cos \chi_{l k} & -\sin \chi_{l k} \\
\sin \chi_{l k} & \cos \chi_{l k}
\end{array}\right]
$$

System (21) can be uncoupled (diagonalized) in a similar way as we have done it with system (3), namely, by applying the transformation $\left[\delta x_{j}, \delta y_{j}\right]^{T}=\sum_{m=1}^{N} e^{i \varphi_{m} j}\left[\delta u_{m}, \delta y_{m}\right]^{T}$ with $\varphi_{m}=2 \pi m / N, m=1, \ldots, N$. As a result, we obtain the uncoupled system

$$
\begin{aligned}
{\left[\begin{array}{c}
\delta u_{m}^{\prime} \\
\delta u_{m}^{\prime}
\end{array}\right]=} & -\left(C_{l k}+a_{l k}^{2}\left[\begin{array}{cc}
1 & 1 \\
1 & 1
\end{array}\right]\right)\left[\begin{array}{l}
\delta u_{m} \\
\delta v_{m}
\end{array}\right] \\
& +e^{i \varphi_{m}} C_{l k}\left[\begin{array}{c}
\delta u_{m}(t-\tau) \\
\delta v_{m}(t-\tau)
\end{array}\right],
\end{aligned}
$$

which leads to the following characteristic equation: 


$$
\operatorname{det}\left(\mu I+a_{l k}^{2}\left[\begin{array}{cc}
1 & 1 \\
1 & 1
\end{array}\right]+C_{l k}\left(1-e^{i \varphi_{m}-\mu \tau}\right)\right)=0
$$

or, equivalently,

$$
\begin{aligned}
0= & \mu^{2}+2 \mu\left[a_{l k}^{2}+\left(1-e^{i \varphi_{m}-\mu \tau}\right) \cos \left(\varphi_{l}-\tau \omega_{l k}\right)\right] \\
& +2 a_{l k}^{2}\left(1-e^{i \varphi_{m}-\mu \tau}\right) \cos \left(\varphi_{l}-\tau \omega_{l k}\right) \\
& +\left(1-e^{i \varphi_{m}-\mu \tau}\right)^{2} .
\end{aligned}
$$

The solutions $\mu$ of this characteristic equation determine the stability of the periodic solution. Let us recall that all periodic solutions, which we consider, are characterized by the two indices $l$ and $k$. The index $1 \leq l \leq N$ stands for the coupling modes and $k$ for the additional delay modes. Thus, by fixing some $k$ and $l$, one fixes a particular periodic pattern [see also Fig. 8]. The index $m$ stands for the different perturbation modes, i.e., solutions of Eq. (22) for all different 1 $\leq m \leq N$ constitute a set of eigenvalues $\mu$ determining the stability of a given pattern. In particular, if all the eigenvalues have negative real parts (except for the trivial one, which is zero and corresponds to the Floquet multiplier 1), then the chosen pattern is asymptotically stable.

\section{Characterizing stable and unstable patterns}

In this section we provide an analysis of the stability of the periodic patterns using the derived characteristic Eq. (22) and compare it with the known cases of a single oscillator with delay and coupled oscillators without delay $[13,56]$. In the Appendix, we derive the characteristic equation for periodic solutions (rotating waves) for the single Stuart-Landau oscillator with delayed feedback of the form

$$
\dot{z}(t)=(\alpha+i \beta) z(t)-z(t)|z(t)|^{2}+z(t-\tau) .
$$

Such periodic solutions have the form

$$
z(t)=a_{k} e^{i \omega_{k} t}
$$

with some amplitude $a_{k}$ and frequency $\omega_{k}$ given in Eqs. (A1) and (A2). Their stability is governed by the characteristic equation (see the Appendix)

$$
\begin{aligned}
0= & \mu^{2}+2 \mu\left[a_{k}^{2}+\left(1-e^{-\mu \tau}\right) \cos \left(\omega_{k} \tau\right)\right] \\
& +2 a_{k}^{2}\left(1-e^{-\mu \tau}\right) \cos \left(\omega_{k} \tau\right)+\left(1-e^{-\mu \tau}\right)^{2}=0 .
\end{aligned}
$$

In the ring of the coupled Stuart-Landau oscillators without delay [13],

$$
\dot{z}_{j}(t)=(\alpha+i \beta) z_{j}(t)-z_{j}(t)\left|z_{j}(t)\right|^{2}+z_{j+1}(t),
$$

the corresponding periodic patterns take form (17). Contrary to the delayed case, the amplitude and frequency are explicitly given by $a_{l}=\sqrt{\alpha+\cos \varphi_{l}}$ and $\omega_{l}=\beta+\sin \varphi_{l}$. Their stability is described by the polynomial characteristic equation,

$$
\begin{aligned}
0= & \mu^{2}+2 \mu\left[a_{l}^{2}+\left(1-e^{i \varphi_{m}}\right) \cos \varphi_{l}\right]+2 a_{l}^{2}\left(1-e^{i \varphi_{m}}\right) \cos \varphi_{l} \\
& +\left(1-e^{i \varphi_{m}}\right)^{2} .
\end{aligned}
$$

It is easy to see that the characteristic equation for the ring of delayed coupled oscillators [Eq. (22)] is generalization of two cases [Eqs. (25) and (27)]. Moreover, as we will see below, the asymptotic cases, where either the delay or the number of oscillators is large, provide the same result concerning the existence and stability of the periodic patterns in all three cases.

Let us start with the Hopf bifurcation condition, where the periodic patterns lose their stability. The Hopf bifurcation condition can be obtained by substituting $\mu=i \Omega$ into the characteristic equation. As a result, we obtain the following conditions:

Case 1 (single delayed oscillator):

$$
\begin{aligned}
0= & -\Omega^{2}+2 i \Omega\left[a_{k}^{2}+\left(1-e^{-i \Omega \tau}\right) \cos \chi_{d}\right] \\
& +2 a_{k}^{2}\left(1-e^{-i \Omega \tau}\right) \cos \chi_{d}+\left(1-e^{-i \Omega \tau}\right)^{2} .
\end{aligned}
$$

Case 2 (coupled oscillators without delay):

$$
\begin{aligned}
0= & -\Omega^{2}+2 i \Omega\left[a_{l}^{2}+\left(1-e^{i \varphi_{m}}\right) \cos \chi_{c}\right]+2 a_{l}^{2}\left(1-e^{i \varphi_{m}}\right) \cos \chi_{c} \\
& +\left(1-e^{i \varphi_{m}}\right)^{2} .
\end{aligned}
$$

Case 3 (delayed coupled oscillators):

$$
\begin{aligned}
0= & -\Omega^{2}+2 i \Omega\left[a_{l k}^{2}+\left(1-e^{i \varphi_{m}-i \Omega \tau}\right) \cos \chi_{c d}\right] \\
& +2 a_{l k}^{2}\left(1-e^{i \varphi_{m}-i \Omega \tau}\right) \cos \chi_{c d}+\left(1-e^{i \varphi_{m}-i \Omega \tau}\right)^{2},
\end{aligned}
$$

where $\varphi_{m}=2 \pi m / N, 1 \leq m \leq N$, and the numbers $l$ and $k$ characterize the chosen pattern. Here, we denoted by $\chi_{d}=\omega_{k} \tau$, $\chi_{c}=\varphi_{l}$, and $\chi_{c d}=\varphi_{l}-\tau \omega_{l k}$ the quantities, which have the same meaning in all cases, i.e., the relative phase differences between neighboring oscillators also taking into account the delay. Now it is easy to see that the only difference between Eqs. (28)-(30) is the phase factor in the exponents. In the case, when the number of oscillators $N$ is large or the delay $\tau$ is large, this factor does not influence the Hopf frequency since $\varphi_{m}$ covers the whole phase range from 0 to $2 \pi$. In particular, it implies that the asymptotic spectrum crosses the imaginary axis at the same Hopf frequencies in all three cases. This, in turn, implies that, intriguingly, the stability properties of the periodic patterns in all three cases are the same.

Thus, we draw here the following important conclusion from the stability analysis: The periodic rotating waves in three different cases of Eqs. (1), (23), and (26) have the same asymptotic stability properties; i.e., if any two patterns are characterized by the same (or nearly the same) phase difference $\chi$ between neighboring oscillators-taking into account also the time delay shift - then they are either stable or unstable simultaneously. In the case of the single oscillator, $\chi$ is just a phase shift due to the delay.

As a result, for the stability of the family of patterns in Eq. (17) one can immediately obtain the Eckhaus bifurcation scenario [13]. This scenario is illustrated in Fig. 9. There, we compare the families of periodic solutions for the above mentioned three cases. The amplitude $a$ (i.e., $a_{l}, a_{k}$, or $a_{k l}$ correspondingly) of solutions is plotted versus parameter $\alpha$. The black line denotes a boundary between stable and unstable periodic solutions (in solid and dotted gray lines, respectively). In particular, all solutions above this line are asymptotically stable, and the solutions below the line are unstable. Obviously, the Eckhaus line is the same for all cases, while the family of periodic solutions may contain 


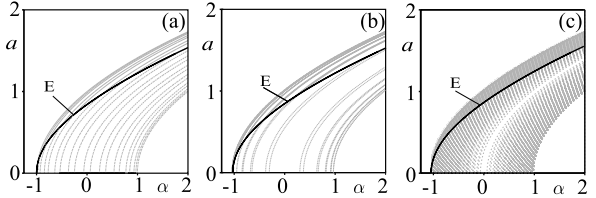

FIG. 9. Periodic patterns and their stability for (a) 20 coupled systems without delay, (b) 1 system with delayed feedback with $\tau$ $=15$, and (c) 20 delayed coupled oscillators with $\tau=15$. The solid black line in each plot shows the stability boundary (above this line periodic solutions are stable).

different numbers of solutions depending on the delay or the size of the array $N$. Finally, we note that the rate of multistability is increased in comparison with the nondelayed case due to the interaction delays.

\section{COUPLED SPIKING NEURONS}

In this section we demonstrate that the phenomena observed in the previous sections for the coupled Stuart-Landau limit-cycle oscillators can be found for a ring of delaycoupled spiking neurons. We consider a system of unidirectionally coupled FHN oscillators $[64,65]$ interacting via excitatory chemical synapses,

$$
\begin{gathered}
\dot{v}_{j}=v_{j}-v_{j}^{3} / 3-w_{j}+I_{j}+C\left(V-v_{j}\right) s_{j+1}(t-\tau), \\
\dot{w}_{j}=0.08\left(v_{j}+0.7-0.8 w_{j}\right), \\
\dot{s}_{j}=0.5\left(1-s_{j}\right) /\left\{1+\exp \left[-4\left(v_{j}-1.5\right)\right]\right\}-0.6 s_{j}, \\
j=1,2, \ldots, N .
\end{gathered}
$$

Variable $v_{j}$ models the membrane potential of a single cell, and $I_{j}$ is a constant current controlling the spiking dynamics of a neuron. In what follows we consider $I_{j}$ being randomly Gaussian distributed around the mean value $\bar{I}=0.4$ with standard deviation $\sigma=0.005$. For such values of $I_{j}$ the timeaveraged spiking frequencies $f_{j}$ (average number of spikes per second) of individual neurons are distributed around the mean frequency $\bar{f} \approx 23.6 \mathrm{~Hz}$ with standard deviation $\sigma_{f}$ $\approx 0.13$ [Fig. 10(a), black circles]. The synaptic coupling between the neurons in ensemble (31) is realized via a postsynaptic potential $s_{j}$ generated by neuron $j[71,72]$. It is modeled in the standard way by an additional equation for $s_{j}(t)$ $[73,74]$. Parameter $C$ defines the coupling strength, $\tau$ is the time delay in coupling, and $V$ is the reversal potential taken as $V=2$ for excitatory coupling. As for the ensemble of the Stuart-Landau limit-cycle oscillators [Eq. (1)], we assume that the neurons are unidirectionally coupled in a ring such that $s_{N+1} \equiv s_{1}$.

We first consider the case of zero delay $\tau=0$. If the coupling strength in neuronal ensemble (31) increases, the neurons synchronize and oscillate with the same frequency [Fig. 10(a)]. Depending on the initial conditions, the frequency of the synchronized states can attain different values indicating a multistability of synchronized regimes. For example, for narrowly distributed initial conditions
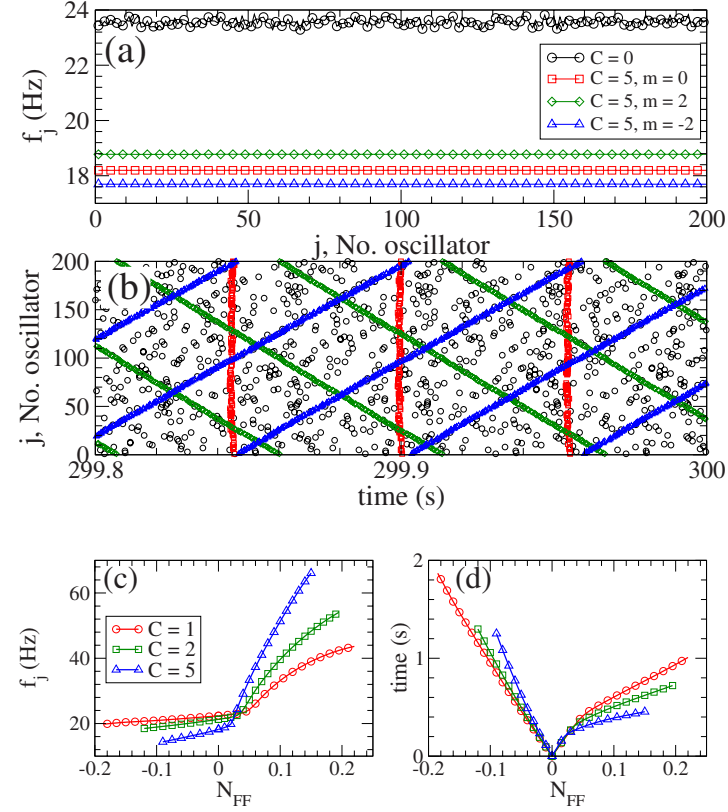

FIG. 10. (Color online) Synchronized spatial dynamics of the unidirectionally synaptically coupled spiking neurons [Eq. (31)]. (a) Individual time-averaged spiking frequencies $f_{j}$ (average number of spikes per second) for the coupling strength $C=5$ and for different initial conditions leading to different numbers of traveling firing fronts on the ring $m=0$ (in-phase synchronization, red squares), $m$ $=2$ (green diamonds), and $m=-2$ (blue triangles) as indicated in the legend. A negative value in the latter case means that the traveling fronts move in the direction opposite to the direction of coupling in the ensemble. Uncoupled neurons $(C=0)$ fire at different spiking frequencies (black circles). (b) Raster plot of the neuronal firing, where the onsets of spikes are indicated by symbols corresponding to those in plot (a) (see legend): asynchronous firing $(C=0$, uniformly distributed black circles), in-phase synchronization $(C=5$, $m=0$, red squares arranged along vertical lines), two firing fronts propagating in the direction of the coupling $(C=5, m=2$, green diamonds arranged along lines with negative slope), and two firing fronts propagating in opposite direction of the coupling $(C=5, \mathrm{~m}$ $=-2$, blue triangles arranged along lines with positive slope). (c) Synchronized spiking frequencies of stable traveling $m$-front solutions versus the relative number $N_{F F}=m / N$ of the firing fronts. The coupling strength is indicated in the legend. (d) Traveling time of a complete rotation of the firing fronts along the ring of neurons. Same coloring as in plot (c). Number of oscillators $N=200$ and delay $\tau=0$.

$\left\{\left[v_{j}(0), w_{j}(0), s_{j}(0)\right]\right\}_{j=1}^{N}$ the oscillators synchronize at the frequency $f_{j} \approx 18.2 \mathrm{~Hz}$ [Fig. 10(a), red squares], whereas for broadly distributed initial condition the synchronized frequency can be $f_{j} \approx 18.8 \mathrm{~Hz}$ or $f_{j} \approx 17.7 \mathrm{~Hz}$ [Fig. 10(a), green diamonds and blue triangles]. To investigate this phenomenon we consider the above initial conditions distributed on a limit cycle $\gamma(t)=[v(t), w(t), s(t)], t \in[0, T]$ of a single FHN neuronal oscillator, where $T$ is a period of oscillations. Following Eq. (17), for a fixed integer $l$ the initial conditions are taken as $\left[v_{j}(0), w_{j}(0), s_{j}(0)\right]=\gamma\left(t_{j}\right)$, where $t_{j}$ $=j \frac{T}{N} l(\bmod T)$. The above synchronized states with different synchronized frequencies are obtained for $l=0,2$, and -2 .

Besides different frequencies, synchronized neurons demonstrate a different spatial organization of the spiking dy- 
namics. For narrowly distributed initial conditions, e.g., for $l=0$, the oscillators are in-phase synchronized and all neurons fire simultaneously [Fig. 10(b), red squares]. On the other hand, for broadly distributed initial conditions the neuronal activity can be organized in a traveling-wave regime having one or several firing fronts traveling along the ring. In such a regime, the next neuron in the ring fires after its neighboring neuron generates a spike. Intriguingly, the firing fronts can move along the ring either in the direction of the synaptic coupling or in the opposite direction. For example, for $l=2$ we obtain two traveling fronts propagating along the ring in the direction of the coupling [Fig. 10(b), green diamonds]. Conversely, for $l=-2$ the two traveling firing fronts move along the ring in opposite direction of the coupling [Fig. 10(b), blue triangles]. We also note that uncoupled and desynchronized neurons do not demonstrate any spatial structure [Fig. 10(b), black circles].

We found that a number of $m$-front solutions can be stable for neuronal ensemble (31) for a range of the firing fronts $m$ [Figs. 10(c) and 10(d)]. For the traveling front dynamics we define the relative number of the firing fronts $N_{F F}=m / N$, where the number of traveling fronts $m$ can be either positive or negative depending on whether the fronts travel along or against the direction of the coupling, respectively. Each of such solutions is characterized by different synchronized spiking frequency $f_{j}=f$, which increases if the number of positive fronts (positive $m$ ) increases and decreases for increasing number of negative fronts (negative $m$ ) [Fig. 10(c)]. Furthermore, the spatial velocity of the front propagation along the network significantly slows down if the number of firing fronts (either positive or negative) increases. It is also worth noting that a stronger coupling in neuronal ensemble (31) without delay appears to counteract the stability of the firing fronts and makes the range of the stable fronts smaller [Figs. 10(c) and 10(d)]. These results agree with those obtained for the relaxation oscillators, such as the Morris-Lecar and the Ellias-Grossberg neuronal models, where strong coupling among neurons support the in-phase synchronization at the expenses of the traveling waves [75].

The situation is different for a finite delay $\tau$ in the coupling between the neurons. Stronger coupling apparently promotes the stability of the traveling firing fronts and extends the range of stable fronts [Fig. 11(a)]. For large delay and coupling neuronal ensemble (31) exhibits multistability of the traveling firing fronts, which becomes more pronounced if the time delay gets larger [Figs. 11(b)-11(f)]. In such a way, several stable $m$-front solutions with the same number $m$ of the firing fronts can coexist. They differ from each other by the spiking frequencies $f_{j}$ of the synchronized neurons and, correspondingly, by the propagation time on the ring. For example, for delay $\tau=30 \mathrm{~ms}$ and coupling $C=5$ and for the range of the relative number of traveling fronts $N_{F F} \in[-0.5,0.135] \cup[0.335,0.5]$ system (31) has two stable $m$-front solutions with the same number $m=N_{F F} N$ (for $N$ $=200, m \in[-100,27] \cup[67,100])$ of the firing fronts but with different spiking frequencies $f_{j}$ of synchronized neurons [Fig. 11(b)]. For the above values of the parameters, the two different stable $m$-front solutions with $m=1$ firing fronts are illustrated in Fig. 11(g) (red and light blue dots). The number of coexisting stable $m$-front solutions with the same number
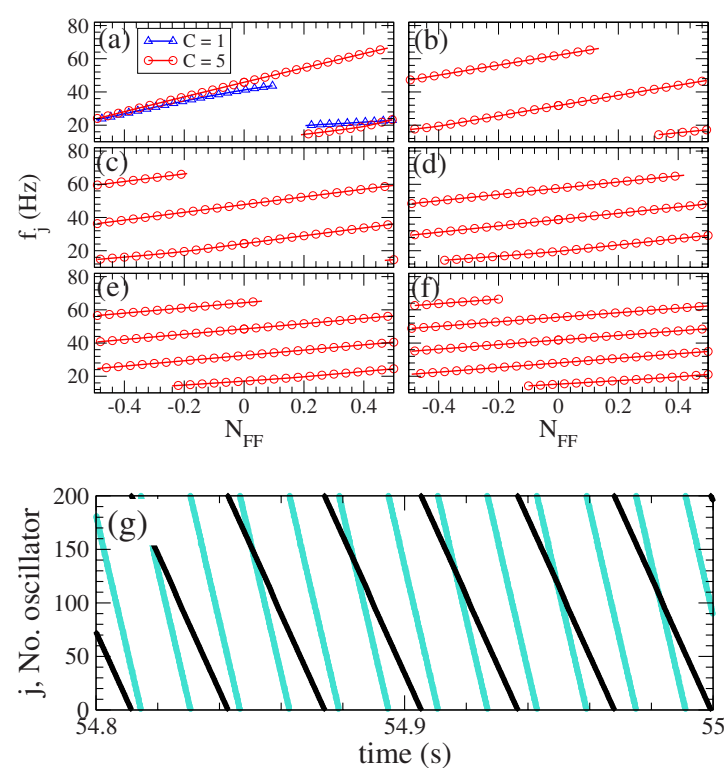

FIG. 11. (Color online) Delay-induced multistability of the traveling firing fronts of the unidirectionally synaptically coupled spiking neurons [Eq. (31)]. (a)-(f) Individual time-averaged spiking frequencies $f_{j}$ (average number of spikes per second) versus the relative number $N_{F F}=m / N$ of the firing fronts for different coupling strength $C$ in (a) as indicated in the legend and delay: (a) $\tau=$ (a) 20, (b) 30, (c) 40, (d) 50, (e) 60, and (f) $70 \mathrm{~ms}$. (g) Raster plot of the neuronal firing, where the onsets of spikes are indicated by dots. For different initial conditions two different coexisting stable solutions can be found (black and light blue dots) of $m=1$ traveling firing fronts. Delay $\tau=30 \mathrm{~ms}$ and the coupling strength $C=5$, which correspond to plot (b). Coupling $C=5$ in (b)-(f), and the number of oscillators (a)-(c), (g) $N=200$, and (d)-(f) $N=100$.

$m=N_{F F} N$ of the firing fronts increases as the time delay in system (31) gets larger. For example, there exist three different stable $m$-front solutions for delay $\tau=40$ and $50 \mathrm{~ms}$ [Figs. $11(\mathrm{c})$ and $11(\mathrm{~d})]$, four different stable $m$-front solutions for delay $\tau=60$ and $70 \mathrm{~ms}$ [Figs. 11(e) and 11(f)], and so on.

The delay-induced multistability of the in-phase synchronized states ( $m=0$ traveling firing fronts) of neuronal ensemble (31) [Fig. 10(b), red squares] can be investigated with the help of a single neuron with delayed feedback, i.e., for $N=1$ in Eq. (31). Indeed, since for small parameter mismatch among the neurons the in-phase synchronization is characterized by almost identical amplitude dynamics of the neurons, they could be replaced by a single neuron with delayed feedback. In this way, the continuation by delay parameter $\tau$ of a single neuron with delayed feedback reveals an interval of bistability of the spiking dynamics for $\tau$ $\in(6.7,10)$ and feedback strength $C=5$ [Fig. 12(a), red circles]. In this parameter interval different initial conditions can lead to either slow [Fig. 12(a), red circles, lower branch] or fast [Fig. 12, red circles, upper branch] spiking dynamics of a neuron. For example, for $\tau=7$ and $C=5$, the FHN neuron with delayed feedback can oscillate with either $f$ $=14 \mathrm{~Hz}$ or $f=96.9 \mathrm{~Hz}$. This dynamics is reflected by a system of $N=200$ identical FHN neurons [Eq. (31)], where the neuronal oscillators synchronize in-phase either at one or another of the above frequencies for a proper choice of the 

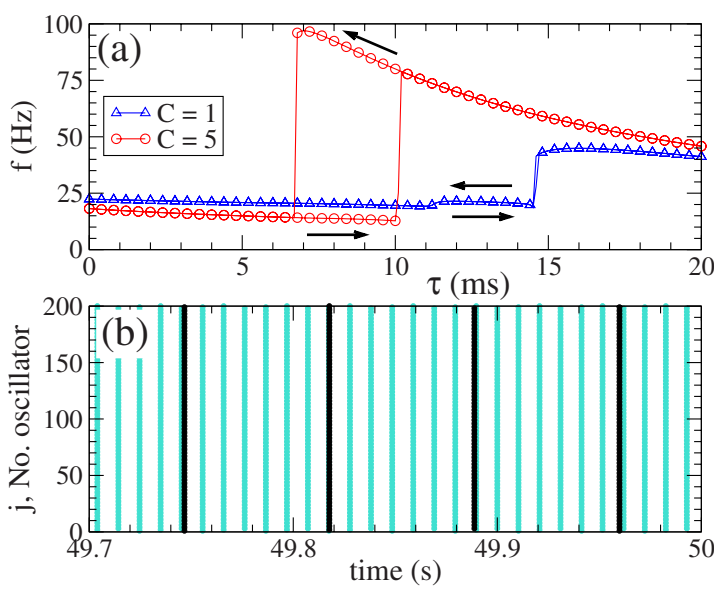

FIG. 12. (Color online) Delay-induced bistability of the in-phase synchronized states of the unidirectionally synaptically coupled spiking neurons [Eq. (31)]. (a) Spiking frequency of a single FHN neuron with delayed feedback, i.e., $N=1$ in system (31), versus time delay $\tau$ for the feedback strength $C$ as indicated in the legend. The direction of continuation by parameter $\tau$ is indicated by arrows. (b) Raster plot of the neuronal firing, where the onsets of spikes are indicated by dots. Two stable coexisting in-phase synchronized states (black and light blue dots) of the ensemble of $N=200$ identical spiking neurons [Eq. (31)] are illustrated for coupling $C=5$ and delay $\tau=7 \mathrm{~ms}$. The constant currents $I_{j}=0.4$.

initial conditions [Fig. 12(b), black and light blue dots]. For a smaller feedback strength $C=1$ the continuation by parameter $\tau$ in both directions gives the same values of the spiking frequency and a bistability region is not visible in the scale of the plot [Fig. 12(a), blue triangles].

Although the multistability of the in-phase synchronized solutions of neuronal ensemble (31) can be found for relatively small values of the time delay (Fig. 12), the long-range multistability of the traveling firing fronts starts at larger delays (Fig. 11). It is interesting to observe that the spiking frequencies $f_{j}$ of the coexisting stable traveling-front solutions are bounded to some frequency interval $f_{j}$ $\in\left[f_{\min }, f_{\max }\right]$, which depends on the coupling strength and can be found already for the delay-free case, i.e., for $\tau=0$. Indeed, the spiking frequencies of the stable $m$-front solutions of neurons [Eq. (31)] coupled without delay $(\tau=0)$ are bounded, for instance, to the interval $f_{j} \in[14 \mathrm{~Hz}, 66 \mathrm{~Hz}]$ for $C=5$ and to the interval $f_{j} \in[20 \mathrm{~Hz}, 44 \mathrm{~Hz}]$ for $C=1$ [Fig. 10 (c)]. This property is preserved if a finite delay is introduced [Fig. 11(a)]. Then, the multistability of the travelingfront solutions is manifested in that the branches of such solutions densely fill the above frequency intervals as the delay in the coupling between the neurons increases [Figs. 11(a)-11(f)]. This is in accordance with the results obtained for the delay-coupled Stuart-Landau limit-cycle oscillators [see Eq. (20)].

\section{CONCLUSIONS}

In summary, the introduction of delays in a ring of coupled oscillators leads to the emergence of additional rotating waves and their multistability. The emergence of rotat- ing waves is mediated by the Hopf bifurcations of the symmetric equilibrium. We have described stability properties of emerging periodic patterns. The phenomena are shown analytically for the Stuart-Landau oscillators as well as numerically for the coupled FitzHugh-Nagumo systems interacting via excitatory chemical synapses. In order to obtain the analytical description of the phenomena, we have extensively used the phase-shift symmetry of the Stuart-Landau oscillators, i.e., the symmetry of the system with respect to the transformation $z \rightarrow e^{i \varphi} z$. This symmetry allows us to obtain the explicit expressions for the rotating waves. The similarity of the results for the FitzHugh-Nagumo and the StuartLandau systems indicate that the described phenomena do not depend on the explicit form or symmetries of the individual oscillator.

In the case when the delay times $\tau_{j}$ are dispersed (not identical), transformation (2) can be used and the corresponding reduced system will have identical delays. While the described spatiotemporal patterns are periodic in time and in space for the case of identical delays, they become irregular and even discontinuous in space (when the spatial variable being considered as the index $j$ ) but still periodic in time for the original system with dispersed delays. Because of the spatial discontinuity of such patterns, it is preferable to speak about spiking patterns rather than spatiotemporal patterns, especially in the case of neurons. It follows from Eq. (2) that by an appropriate choice of delays $\tau_{j}$, any timeperiodic spiking pattern can be realized with the restriction that each oscillator has one maximum per period (or each neuron fires once per period). In a forthcoming publication we will extend this aspect and provide a more detailed discussion.

The results obtained in this study are also relevant from a neuroscientific standpoint. To reveal the directionality of interaction within loops of neurons or neuronal populations, experimentalists often infer the directionality of interaction from the sequence of activation or firing (e.g., $[76,77])$ : A sequential activation is typically interpreted as being caused by a directional coupling in the sense that neurons that fire later are driven by those which fire earlier. Our study nicely illustrates the rich and-from the classical experimentalist's standpoint — puzzling situation that the directionality of interaction need not at all correspond to the timing pattern of the units forming the loop. Although in our model there is a purely unidirectional coupling, the neurons may fire coincidently or they may fire one after the other in sequence, corresponding to the directionality of coupling. Moreover, intriguingly, they may even fire in reverse order, i.e., in a timing sequence completely opposite to the directionality of coupling. This nicely illustrates that the standard procedure, used by experimentalists, to identify timing sequences with coupling patterns may actually lead to enormous artifacts.

\section{ACKNOWLEDGMENTS}

P.P. and S.Y. acknowledge the support of DFG Research Center Matheon "Mathematics for key technologies" under Project No. D21. P.P. acknowledges the support of Department of International Cooperation of Poland under Project 
No. DWM/N97/DAAD/2008 and from Foundation for Polish Science (the START Foundation).

\section{APPENDIX: CHARACTERISTIC EQUATION FOR ROTATING WAVES IN THE SINGLE STUART-LANDAU OSCILLATOR WITH DELAYED FEEDBACK}

The equation for a single Stuart-Landau oscillator with delayed feedback is given in Eq. (23). Properties of the periodic patterns in this system have been considered in [56] in the limit of large delay by transforming it to the complex Ginzburg-Landau equation. Here, we derive the corresponding characteristic equation directly for Eq. (23).

First of all, periodic solutions of Eq. (23) can be obtained by substituting

$$
z(t)=a e^{i \omega t}
$$

into Eq. (23). The resulting equations can be solved with respect to the amplitude and frequency,

$$
\begin{gathered}
a_{k}=\sqrt{\alpha+\cos \left(\omega_{k} \tau\right),} \\
\omega_{k}=\beta-\sin \left(\omega_{k} \tau\right),
\end{gathered}
$$

where index $k$ corresponds to different solutions of transcendental Eq. (A2) for given $\tau, \alpha$, and $\beta$.

In order to find the stability of the obtained periodic solutions $a_{k} e^{i \omega_{k} t}$, we linearize system (23) around these solutions. For this, we first make the transformation in Eq. (23) by introducing new variables $x(t)$ and $y(t)$,

$$
z(t)=a_{k} e^{i \omega_{k} t}[x(t)+i y(t)],
$$

which leads to the following system with respect to $x(t)$ and $y(t)$ :

$$
\begin{aligned}
\dot{x}(t)= & \alpha x(t)-\left(\beta-\omega_{k}\right) y(t)-x(t)\left[x^{2}(t)+y^{2}(t)\right] \\
& +x(t-\tau) \cos \left(\omega_{k} \tau\right)+y(t-\tau) \sin \left(\omega_{k} \tau\right), \\
\dot{y}(t)= & \alpha y(t)+\left(\beta-\omega_{k}\right) y(t)-y(t)\left[x^{2}(t)+y^{2}(t)\right] \\
& -x(t-\tau) \sin \left(\omega_{k} \tau\right)+y(t-\tau) \cos \left(\omega_{k} \tau\right) .
\end{aligned}
$$

In the new system, the periodic solution $z(t)=a_{k} e^{i \omega_{k} t}$ is reduced to the family of equilibria $x+i y=e^{i \kappa}, \kappa \in \mathbb{R}$. In order to find its stability, it is necessary to linearize Eq. (A3) around any of these equilibria. We choose $x=y=1 / \sqrt{2}$. The corresponding linearized system in the vector form reads as follows:

$$
\left[\begin{array}{l}
\delta x^{\prime}(t) \\
\delta y^{\prime}(t)
\end{array}\right]=-\left(C_{k}+a_{k}\left[\begin{array}{ll}
1 & 1 \\
1 & 1
\end{array}\right]\right)\left[\begin{array}{l}
\delta x(t) \\
\delta y(t)
\end{array}\right]+C_{k}\left[\begin{array}{l}
\delta x(t-\tau) \\
\delta y(t-\tau)
\end{array}\right],
$$

where

$$
C_{k}=\left[\begin{array}{cc}
\cos \left(\omega_{k} \tau\right) & \sin \left(\omega_{k} \tau\right) \\
-\sin \left(\omega_{k} \tau\right) & \cos \left(\omega_{k} \tau\right)
\end{array}\right] .
$$

In order to obtain Eq. (A4), we used the expressions in Eqs. (A1) and (A2). The characteristic equation is obtained from Eq. (A4) by substituting $\delta x(t)=e^{\mu t}$,

$$
\operatorname{det}\left(\mu I+a_{k}^{2}\left[\begin{array}{ll}
1 & 1 \\
1 & 1
\end{array}\right]+C_{k}\left(1-e^{-i \mu \tau}\right)\right)=0 .
$$

Finally, we obtain

$$
\begin{aligned}
\mu^{2}+ & 2 \mu\left[a_{k}^{2}+\left(1-e^{-\mu \tau}\right) \cos \psi_{\tau}\right]+2 a_{k}^{2}\left(1-e^{-\mu \tau}\right) \cos \psi_{\tau} \\
& +\left(1-e^{-\mu \tau}\right)^{2}=0,
\end{aligned}
$$

where $\psi_{\tau}=\omega_{k} \tau$.
[1] S. H. Strogatz, Nature (London) 410, 268 (2001).

[2] R. Zillmer, R. Livi, A. Politi, and A. Torcini, Phys. Rev. E 74, 036203 (2006).

[3] E. Mosekilde, Y. Maistrenko, and D. Postnov, Chaotic Synchronization. Application to Living Systems (World Scientific, Singapore, 2002).

[4] C. Hauptmann, O. Popovych, and P. A. Tass, Biol. Cybern. 93, 463 (2005).

[5] A. Tsonis and P. Roebber, Physica A: Statistical and Theoretical Physics 333, 497 (2004).

[6] A. Pikovsky, M. Rosenblum, and J. Kurths, Synchronization: A Universal Concept in Nonlinear Sciences (Cambridge University Press, Cambridge, 2001).

[7] P. Tass, Phase Resetting in Medicine and Biology: Stochastic Modelling and Data Analysis, Springer Series in Synergetics (Springer, Berlin, 1999).

[8] H. Daido and K. Nakanishi, Phys. Rev. E 75, 056206 (2007).

[9] K. Kaneko, Physica D 41, 137 (1990).

[10] S. Yanchuk, Y. Maistrenko, and E. Mosekilde, Math. Comput. Simul. 54, 491 (2001).

[11] V. A. Makarov, E. del Río, W. Ebeling, and M. G. Velarde,
Phys. Rev. E 64, 036601 (2001).

[12] P. G. Lind, J. Corte-Real, and J. A. C. Gallas, Phys. Rev. E 69, 066206 (2004).

[13] S. Yanchuk and M. Wolfrum, Phys. Rev. E 77, 026212 (2008).

[14] Y. Kuramoto, Chemical Oscillations, Waves, and Turbulence (Springer, Berlin, 1984).

[15] D. M. Abrams and S. H. Strogatz, Phys. Rev. Lett. 93, 174102 (2004).

[16] C.-U. Choe, T. Dahms, P. Hövel, and E. Schöll, Phys. Rev. E 81, 025205 (2010).

[17] J. G. Restrepo, E. Ott, and B. R. Hunt, Phys. Rev. Lett. 93, 114101 (2004).

[18] A. Arenas, A. Díaz-Guilera, J. Kurths, Y. Moreno, and C. Zhou, Phys. Rep. 469, 93 (2008).

[19] O. D'Huys, R. Vicente, T. Erneux, J. Danckaert, and I. Fischer, Chaos 18, 037116 (2008).

[20] A. Takamatsu, R. Tanaka, H. Yamada, T. Nakagaki, T. Fujii, and I. Endo, Phys. Rev. Lett. 87, 078102 (2001).

[21] P. Woafo and H. G. E. Kadji, Phys. Rev. E 69, 046206 (2004).

[22] M. Bonnin, Physica D 238, 77 (2009).

[23] W. Zou and M. Zhan, SIAM J. Appl. Dyn. Syst. 8, 1324 
(2009).

[24] I. Waller and R. Kapral, Phys. Rev. A 30, 2047 (1984).

[25] H. Daido, Phys. Rev. Lett. 78, 1683 (1997).

[26] P. C. Bressloff, S. Coombes, and B. de Souza, Phys. Rev. Lett. 79, 2791 (1997).

[27] R. Dodla, A. Sen, and G. L. Johnston, Phys. Rev. E 69, 056217 (2004).

[28] I. P. Mariño, V. Pérez-Muñuzuri, V. Pérez-Villar, E. Sánchez, and M. A. Matías, Physica D 128, 224 (1999).

[29] G. Van der Sande, M. C. Soriano, I. Fischer, and C. R. Mirasso, Phys. Rev. E 77, 055202 (2008).

[30] Y. Zhang, G. Hu, and H. A. Cerdeira, Phys. Rev. E 64, 037203 (2001).

[31] X. L. Deng and H. B. Huang, Phys. Rev. E 65, 055202 (2002).

[32] M. A. Matías and J. Guiémez, Phys. Rev. Lett. 81, 4124 (1998).

[33] K. Pakdaman, C. P. Malta, C. Grotta-Ragazzo, O. Arino, and J.-F. Vibert, Phys. Rev. E 55, 3234 (1997).

[34] Y. Horikawa and H. Kitajima, Physica D 238, 216 (2009).

[35] S. J. Guo and L. H. Huang, Acta Mathematica Sinica 23, 799 (2007).

[36] F. Pasemann, Neural Networks 8, 421 (1995).

[37] P. Perlikowski, S. Yanchuk, M. Wolfrum, A. Stefanski, P. Mosiolek, and T. Kapitaniak, Chaos 20, 013111 (2010).

[38] P. Baldi and A. Atiya, IEEE Trans. Neural Netw. 5, 612 (1994).

[39] M. Golubitsky, I. Stewart, P. Buono, and J. Collins, Nature (London) 401, 693 (1999).

[40] H. Bergman, A. Feingold, A. Nini, A. Raz, H. Slovin, M. Abeles, and E. Vaadia, Trends Neurosci. 21, 32 (1998).

[41] H. Erzgräber, B. Krauskopf, and D. Lenstra, SIAM J. Appl. Dyn. Syst. 5, 30 (2006).

[42] T. W. Carr, I. B. Schwartz, M.-Y. Kim, and R. Roy, SIAM J. Appl. Dyn. Syst. 5, 699 (2006).

[43] I. Fischer, R. Vicente, J. M. Buldú, M. Peil, C. R. Mirasso, M. C. Torrent, and J. Garcia-Ojalvo, Phys. Rev. Lett. 97, 123902 (2006).

[44] T. Heil, I. Fischer, W. Elsäßer, J. Mulet, and C. R. Mirasso, Phys. Rev. Lett. 86, 795 (2001).

[45] R. Vicente, I. Fischer, and C. R. Mirasso, Phys. Rev. E 78, 066202 (2008).

[46] H.-J. Wünsche et al., Phys. Rev. Lett. 94, 163901 (2005).

[47] A. L. Franz, R. Roy, L. B. Shaw, and I. B. Schwartz, Phys. Rev. E 78, 016208 (2008).

[48] C. Hauptmann, O. Omel'chenko, O. V. Popovych, Y. Maistrenko, and P. A. Tass, Phys. Rev. E 76, 066209 (2007).

[49] C. Masoller, M. C. Torrent, and J. Garcia-Ojalvo, Phys. Rev. E 78, 041907 (2008).

[50] E. Rossoni, Y. Chen, M. Ding, and J. Feng, Phys. Rev. E 71, 061904 (2005).

[51] M. Dhamala, V. K. Jirsa, and M. Ding, Phys. Rev. Lett. 92, 074104 (2004).
[52] H. G. Schuster and P. Wagner, Prog. Theor. Phys. 81, 939 (1989).

[53] S. Yanchuk, Phys. Rev. E 72, 036205 (2005).

[54] J. Kestler, W. Kinzel, and I. Kanter, Phys. Rev. E 76, 035202 (2007).

[55] M. G. Rosenblum, A. S. Pikovsky, and J. Kurths, Phys. Rev. Lett. 78, 4193 (1997).

[56] M. Wolfrum and S. Yanchuk, Phys. Rev. Lett. 96, 220201 (2006).

[57] G. C. Sethia, A. Sen, and F. M. Atay, Phys. Rev. Lett. 100, 144102 (2008).

[58] U. Ernst, K. Pawelzik, and T. Geisel, Phys. Rev. E 57, 2150 (1998).

[59] M. G. Rosenblum and A. S. Pikovsky, Phys. Rev. Lett. 92, 114102 (2004)

[60] O. V. Popovych, C. Hauptmann, and P. A. Tass, Phys. Rev. Lett. 94, 164102 (2005).

[61] O. V. Popovych, C. Hauptmann, and P. A. Tass, Biol. Cybern. 95, 69 (2006).

[62] O. V. Popovych and P. A. Tass, Phys. Rev. E 82, 026204 (2010).

[63] M. Y. Choi, H. J. Kim, D. Kim, and H. Hong, Phys. Rev. E 61, 371 (2000).

[64] R. FitzHugh, Biophys. J. 1, 445 (1961).

[65] J. Nagumo, S. Arimoto, and S. Yoshizawa, Proc. IRE 50, 2061 (1962).

[66] E. M. Izhikevich, IEEE Trans. Neural Netw. 15, 1063 (2004).

[67] L. M. Pecora and T. L. Carroll, Phys. Rev. Lett. 80, 2109 (1998).

[68] P. Hövel and E. Schöll, Phys. Rev. E 72, 046203 (2005).

[69] S. Yanchuk, M. Wolfrum, P. Hövel, and E. Schöll, Phys. Rev. E 74, 026201 (2006).

[70] M. Lichtner, M. Wolfrum, and S. Yanchuk, The Spectrum Of Delay Differential Equations with Large Delay (DFG Research Center Matheon, Berlin, 2009).

[71] W. Gerstner and W. M. Kistler, Spiking Neuron Models: Single Neurons, Populations, Plasticity (Cambridge University Press, Cambridge, 2002).

[72] E. M. Izhikevich, Dynamical Systems in Neuroscience: The Geometry of Excitability and Bursting (MIT Press, Cambridge, Mass., 2007).

[73] D. Golomb and J. Rinzel, Phys. Rev. E 48, 4810 (1993).

[74] D. Terman, J. E. Rubin, A. C. Yew, and C. J. Wilson, J. Neurosci. 22, 2963 (2002).

[75] D. Somers and N. Kopell, Biol. Cybern. 68, 393 (1993).

[76] J. Volkmann, M. Joliot, A. Mogilner, A. A. Ioannides, F. Lado, E. Fazzini, U. Ribary, and R. Llinás, Neurology 46, 1359 (1996).

[77] J. Gross, J. Kujala, M. Hamalainen, L. Timmermann, A. Schnitzler, and R. Salmelin, Proc. Natl. Acad. Sci. U.S.A. 98, 694 (2001). 